\title{
Pleural changes in patients with pneumothoraces and Marfan syndrome
}

\author{
Takako Matsumoto ${ }^{1}$, Kenta Uto $^{2}$, Hideaki Oda $^{2}$, Tamami Isaka ${ }^{1}$, Yoji Nagashima ${ }^{3}$, Masato Kanzaki ${ }^{1}$ \\ ${ }^{1}$ Department of Thoracic Surgery, ${ }^{2}$ Department of Pathology, ${ }^{3}$ Department of Surgical Pathology, Tokyo Women's Medical University, Tokyo, Japan \\ Contributions: (I) Conception and design: T Matsumoto, H Oda, M Kanzaki; (II) Administrative support: T Matsumoto, T Isaka; (III) Provision of \\ study materials or patients: K Uto, H Oda, Y Nagashima, M Kanzaki; (IV) Collection and assembly of data: T Matsumoto, T Isaka; (V) Data analysis \\ and interpretation: T Matsumoto, K Uto; (VI) Manuscript writing: All authors; (VII) Final approval of manuscript: All authors. \\ Correspondence to: Masato Kanzaki. 8-1 Kawada-cho, Shiunjuku-ku, Tokyo 162-8666, Japan. Email: kanzaki.masato@twmu.ac.jp.
}

\begin{abstract}
Background: Patients with Marfan syndrome (MFS) often develop pneumothorax, but the features of pneumothorax in the context of MFS have not been well described in the literature. We clarified the clinical and histopathological characteristics of this condition in these patients.

Methods: Patients with MFS were selected from among all patients who underwent surgery for pneumothorax, between December 1991 and January 2015, in our hospital. We studied the histopathological characteristics of the resected lungs as well as the clinical features of the selected patients, including surgical findings and postoperative recurrence status.

Results: There were 966 operations underwent pneumothorax-related surgeries in our hospital. A total of 16 operations $(1.66 \%)$ were performed on patients with MFS in 11 cases. In this study, 9 patients (6 men, 3 women) were included. Clinically, 7 patients $(77.8 \%)$ had bilateral pneumothoraces and $4(44.4 \%)$ exhibited postoperative recurrent pneumothoraces. Pathologically, the resected pulmonary bullae exhibited blood vessel cystic medial degeneration (55.6\% of cases), calcification (55.6\% of cases), and demonstrated elastic fiber fragmentation and degeneration (all cases).

Conclusions: As in few previous reports, many patients with MFS develop bilateral or postoperative recurrent pneumothoraces. In many patients, characteristic changes in the pulmonary bullae, possibly caused by degenerated elastic fibers, were observed.
\end{abstract}

Keywords: Marfan syndrome (MFS); pneumothorax; bulla; elastic fibers

Submitted Feb 18, 2020. Accepted for publication Jul 18, 2020.

doi: $10.21037 /$ jtd-20-926

View this article at: http://dx.doi.org/10.21037/jtd-20-926

\section{Introduction}

Pneumothorax is a very common pleural disease, with most patients having primary spontaneous pneumothorax (PSP) (1). One of the underlying diseases associated with secondary pneumothorax is Marfan syndrome (MFS), which has an incidence of 1 in every 9,800 births, irrespective of race or sex. Among patients with MFS, $25 \%$ of the cases may be caused by new point mutations in the genes responsible for encoding fibrillin, a protein component of elastic fiber-associated microfibrils $(2,3)$. Thus, MFS is a systemic connective tissue disease caused by mutations in the fibrillin-1 gene (identified in 1991) and the transforming growth factor-beta receptor type 2 gene (identified in 2004) (4). Mutations in these genes may lead to functional impairment of the transforming growth factor-beta signaling pathway and to elastic fiber structural abnormalities that cause connective tissue weakness. This weakness results in various symptoms, such as abnormalities of the skeleton, connective tissue, and eyes (4-6). In the 1996 Ghent criteria for diagnosing MFS, pulmonary pneumothorax and cysts were included as minor criteria. In the revised, 2010 Ghent criteria, the presence of pneumothorax was assigned a systemic score of 2 (7), in recognition of pneumothorax 
being caused by connective tissue abnormalities in patients with MFS (7). The incidence of pneumothorax in patients with MFS is $4-11 \%$, which is hundreds of times higher than that of PSP $(8,9)$. The histopathological characteristics of patients with MFS and pneumothorax have rarely been reported (8-10). Many patients with MFS and cardiovascular complications have been treated in our hospital. Therefore, despite the rarity of the disease, we were able to perform a study to examine the clinical characteristics of patients with MFS-related pneumothoraces and determine the pathological changes, associated with connective tissue abnormalities, in resected pulmonary tissue.

We present the following article in accordance with the STROBE reporting checklist (Available at http://dx.doi. org/10.21037/jtd-20-926).

\section{Methods}

Our retrospective chart review was approved by the ethics committee of the Tokyo Women's Medical University (\#4052). In this study, patients provided informed consent by having the option to opt-out of participation; the study complied with the standards established in the Declaration of Helsinki (as revised in 2013). The inclusion criteria limited participants to those who had undergone pneumothorax surgery at our facility, between January 1991 and December 2015, and who had been diagnosed with MFS according to the revised Ghent criteria. Patients were excluded if they did not undergo surgery and received only medical treatment, if tissue was obtained only by ligation and not by surgery, and if they did not consent to the study.

We examined patient age at the time of surgery, pulmonary cyst locations, incidence of postoperative recurrent pneumothorax, and incidence of bilateral pneumothoraces. All resected specimens were stained with hematoxylin and eosin (HE), elastica van Gieson (EVG), and Alcian blue. Mounted specimens were microscopically observed at $40 \times$ and $100 \times$ magnification. At $40 x$, the presence or absence of calcification, elastic fiber degeneration, and the presence of large blood vessels could be determined, whereas at $100 \times$, the presence or absence of cystic medial degeneration in the blood vessels could be observed. Alcian blue staining was used to observe the presence or absence of mucopolysaccharide deposition in the blood vessels and to facilitate searching for cystic medial degeneration.

Patients who underwent multiple operations were classified according to their age at the time of the first operation at our institution; the pathological specimens were also obtained at that time.

\section{Results}

Between January 1991 and December 2015, 966 operations underwent pneumothorax-related surgeries in our hospital, including 11 ( 8 men and 3 women) who met the revised Ghent diagnostic criteria for MFS (7). One patient had undergone 4 operations, and two patients had undergone 2 operations in our hospital. For two patients, resected specimens were not obtained because the patients underwent ligation or suturing surgeries; they were excluded from this study. Thus, the study group comprised 9 patients (6 men and 3 women); pulmonary bullae specimens, resected from these individuals, were examined histopathologically.

\section{Clinical assessment}

As shown in Table 1, the mean patient age at the time of the initial surgery was 24.6 years. Of the 9 patients, 7 (77.8\%) had bilateral pneumothoraces and 4 (44.4\%) had postoperative recurrent pneumothoraces at the surgical sites. Bullae were found in the apices of the upper lung lobes (a location similar to those associated with patients with PSPs) in all patients and in the superior segment (S6) of the lower lobe in 1 patient. Except for two patients, most patients were included following their first surgery on the affected side. The two patients who underwent their second surgeries also exhibited bullae at the apex of the lung, as in the other cases.

\section{Histopathological assessment}

As shown in Table 2, 5 (55.6\%) of the 9 patients demonstrated blood vessel wall thickening in or near the pulmonary cysts. In these blood vessels, the smooth muscle cell layers were disordered, and cystic medial degeneration was evident (Figure 1). The cystic medial degeneration resembled the characteristic findings of the aortas in patients with MFS. In 5 (55.6\%) of the 9 patients, calcification was present in the cyst walls (Figure 2). Further, all patients demonstrated degenerated, fragmented, or missing elastic fibers in the EVG-stained lung cysts (Figure 3).

\section{Discussion}

We thought that patients with pneumothoraces and MFS 
Table 1 Clinical characteristics of patients with pneumothoraces and Marfan syndrome

\begin{tabular}{lcccccc}
\hline Case No. & Age (years) & Sex & Lung & Location of pulmonary bullae & Number of operations ${ }^{*}$ & Postoperative recurrence \\
\hline 1 & 13 & Male & Right & Apex & Right 1, Left 1 & No \\
2 & 20 & Male & Right & Apex & Right 1, Left 1 & No \\
3 & 21 & Male & Left & Apex & Right 2, Left 3 & Yes \\
4 & 22 & Male & Left & Apex & Right 1, Left 2 & Yes \\
5 & 24 & Female & Right & Apex & Right 2, Left 2 & Yes \\
6 & 25 & Female & Right & Apex & Right 5, Left 1 & Yes \\
7 & 29 & Male & Right & Apex, S & Right 2, Left 1 & No \\
9 & 31 & Male & Right & Apex & Right 1 & Right 1 \\
\hline
\end{tabular}

${ }^{*}$, number of pneumothorax operations. S, segment.

Table 2 Pathological characteristics of specimens recovered from the lungs of patients with pneumothoraces and Marfan syndrome

\begin{tabular}{lccc}
\hline Case No. & Cystic medial degeneration & Calcification & Fragmented and degenerated elastic fibers \\
\hline 1 & No & No & Yes \\
2 & No & No & Yes \\
3 & No & Yes & Yes \\
4 & Yes & No & Yes \\
5 & Yes & Yes & Yes \\
6 & Yes & Yes & Yes \\
7 & Yes & Yes & Yes \\
8 & Yes & Yes & Yes \\
9 & No & No & Yes \\
\hline
\end{tabular}
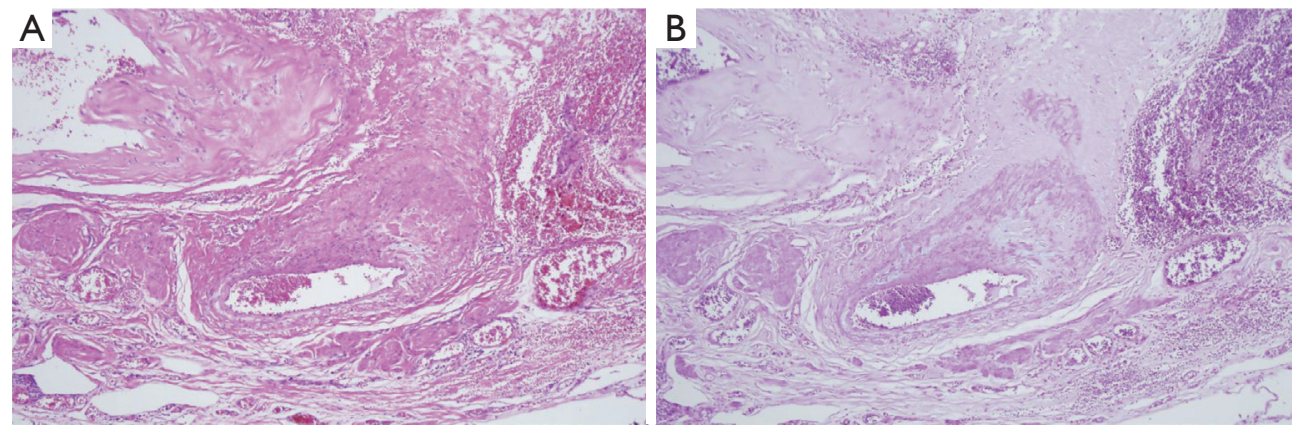

Figure 1 Cystic medial degeneration of the pulmonary vessels. (A) Hematoxylin and eosin stain, $\times 100$; (B) Alcian blue stain, $\times 100$. 


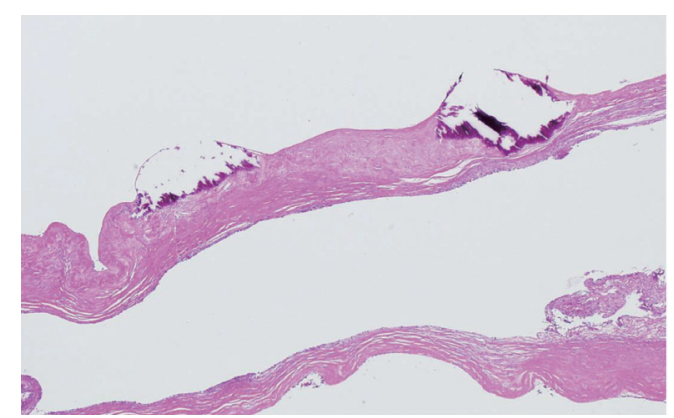

Figure 2 Focal calcification in a pulmonary cyst. Hematoxylin and eosin stain, $\times 40$.

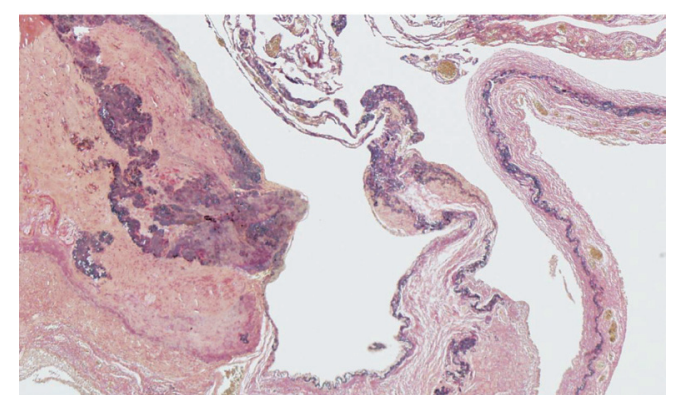

Figure 3 Irregularly thickened, wavy, fragmented, and disconnected elastic fibers present in a pulmonary cyst. Elastica van Gieson stain, $\times 40$.

should have clinical features and pathological features associated with connective tissue abnormalities. However, confirmatory studies have not previously been possible because of the small number of patients. During this study, patients with MFS accounted for $1.66 \%$ of those who underwent pneumothorax-related surgeries in our hospital. This high percentage may be related to the fact that we have treated the cardiovascular abnormalities of many patients with MFS at our hospital.

Our MFS patients also demonstrated high incidences of recurrent and bilateral pneumothoraces. In the present study, as in previous studies involving patients with MFS, the bullae were present in the apices of the lungs $(8,10)$; this is also a finding common to patients with PSP. Lymphangioleiomyomatosis, arising from mutations in either the tuberous sclerosis-1 or tuberous sclerosis-2 genes, is characterized by diffuse lung cysts (11-13). Birt-HoggDubé (BHD) syndrome, which occurs because of folliculin gene mutations, is characterized by multiple cysts in the lungs and its lobes (14-17). Thus, the locations of the lung bullae in patients with lymphangioleiomyomatosis or BHD syndrome differ from those of our patients with MFS. This result suggests different causes for the lung cysts in patients with different diseases. Notably, the locations of the lung bullae in patients with MFS were similar to those in patients with PSP.

Elastic fibers are produced during the early neonatal period and are usually produced rarely, thereafter. Elastin, a major component of medial elastic fibers, levels decrease with age (18). Although our patients were young (mean age, 24.6 years), their elastic fibers were degenerated, fragmented, or had entirely disappeared. In patients with MFS, the alveolar elastic fibers are reportedly irregularly thickened, twisted, fragmented, and blocked (19). In our patients, similar degenerative changes were also observed. In patients with MFS, characteristic changes, e.g., the fragmentation of elastin fibers and the disruption of their arrangement in the aorta, decreased numbers of smooth muscle cells, and deposition of collagen and glycosaminoglycans in the medial cells, are known as cystic medial degeneration. This alteration can be seen in elderly patients as well as in those with MFS (20-24). The fact that similar blood vessel wall changes were found in the resected lung specimens of our patients is noteworthy. The lungs are supplied by the pulmonary and bronchial arteries, whereas the pleura is supplied only by the bronchial arteries (25). However, a conclusive determination of which arteries have been changed cannot be made.

Partial calcification of the lung bullae was seen in many of our patients. In the old Ghent criteria, mitral annulus calcification in patients $<40$ years old was included as a diagnostic factor (26); in the revised criteria, it was removed. There are also reports that elastin fragments cause aortic calcification in MFS (27). Generally, in many tissues, calcification occurs in the elderly. If fragmented elastic fibers cause calcification in young people with MFS, such elastic fibers are also present in lung bullae, which might be the reason lung bullae often calcify. Previous studies have not reported the pulmonary cyst changes, particularly the cystic medial degeneration, which were seen in our patients with MFS (28). These phenomena may be caused by degenerated elastic fibers as well as calcifications. The histopathological characteristics observed in the patients in the present study might present an opportunity for diagnosing patients with MFS in the absence of a family history of the disease.

There are some limitations associated with the current research. The study was a retrospective chart review, not limited to first surgeries, and included older specimens. In addition, we did not examine tissue differences between 
specimens collected during the first and subsequent operations. In the future, we would like to accumulate cases to compare patients with PSP and those with MFS; specimen differences between the first and subsequent surgeries would be analyzed.

\section{Conclusions}

In the present study, we examined the clinical and histopathological characteristics of pneumothorax in patients with MFS. Many patients with MFS had bilateral and postoperative recurrent pneumothoraces, with characteristic changes in the pulmonary bullae. The results of this study may improve the diagnosis of patients with potential sporadic MFS. And we hope that this report will lead to studies to predict the recurrence of pneumothorax.

\section{Acknowledgements}

Funding: None.

\section{Footnote}

Reporting Checklist: The authors have completed the STROBE reporting checklist. Available at http://dx.doi. org/10.21037/jtd-20-926

Data Sharing Statement: Available at http://dx.doi. org/10.21037/jtd-20-926

Conflicts of Interest: All authors have completed the ICMJE uniform disclosure form (available at http://dx.doi. org/10.21037/jtd-20-926). The authors have no conflicts of interest to declare.

Ethical Statement: The authors are accountable for all aspects of the work in ensuring that questions related to the accuracy or integrity of any part of the work are appropriately investigated and resolved. Our retrospective chart review was approved by the ethics committee of the Tokyo Women's Medical University (\#4052). In this study, patients provided informed consent by having the option to opt-out of participation; the study complied with the standards established in the Declaration of Helsinki (as revised in 2013).

Open Access Statement: This is an Open Access article distributed in accordance with the Creative Commons Attribution-NonCommercial-NoDerivs 4.0 International License (CC BY-NC-ND 4.0), which permits the noncommercial replication and distribution of the article with the strict proviso that no changes or edits are made and the original work is properly cited (including links to both the formal publication through the relevant DOI and the license). See: https://creativecommons.org/licenses/by-nc-nd/4.0/.

\section{References}

1. Fujino S, Inoue S, Tezuka N, et al. Physical development of surgically treated patients with primary spontaneous pneumothorax. Chest 1999;116:899-902.

2. Gray JR, Bridges A, Faed M, et al. Ascertainment and severity of Marfan syndrome in a Scottish population. J Med Genet 1994;31:51-4.

3. Judge DP, Dietz HC. Marfan's syndrome. Lancet 2005;366:1965-76.

4. Robinson PN, Arteaga-Solis E, Baldock C, et al. The molecular genetics of Marfan syndrome and related disorders. J Med Genet 2006;43:769-87.

5. Gelb BD. Marfan's syndrome and related disordersmore tightly connected than we thought. $\mathrm{N}$ Engl J Med 2006;355:841-4.

6. Kaartinen V, Warburton D. Fibrillin controls TGF- $\beta$ activation. Nat Genet. 2003;33:331-2.

7. Loeys BL, Dietz HC, Braverman AC, et al. The revised Ghent nosology for the Marfan syndrome. J Med Genet 2010;47:476-85.

8. Karpman C, Aughenbaugh GL, Ryu JH. Pneumothorax and bullae in Marfan syndrome. Respiration 2011;82:219-24.

9. Wood JR, Bellamy D, Child AH, et al. Pulmonary disease in patients with Marfan syndrome. Thorax 1984;39:780-4.

10. Hall JR, Pyeritz RE, Dudgeon DL, et al. Pneumothorax in the Marfan syndrome: prevalence and therapy. Ann Thorac Surg 1984;37:500-4.

11. Seyama K, Kumasaka T, Kurihara M, et al. Lymphangioleiomyomatosis: a disease involving the lymphatic system. Lymphat Res Biol 2010;8:21-31.

12. Tobino K, Johkoh T, Fujimoto K, et al. Computed tomographic features of lymphangioleiomyomatosis: evaluation in 138 patients. Eur J Radiol 2015;84:534-41.

13. Johnson SR, Cordier J, Lazor R, et al. European Respiratory Society guidelines for the diagnosis and management of lymphangioleiomyomatosis. Eur Respir J 2010;35:14-26.

14. Furuya M, Nakatani Y. Birt-Hogg-Dubé syndrome: 
clinicopathological features of the lung. J Clin Pathol 2013;66:178-86.

15. Tobino K, Hirai T, Johkoh T, et al. Differentiation between Birt-Hogg-Dubé syndrome and lymphangioleiomyomatosis: quantitative analysis of pulmonary cysts on computed tomography of the chest in 66 females. Eur J Radiol 2012;81:1340-6.

16. Fabre A, Borie R, Debray MP, et al. Distinguishing the histological and radiological features of cystic lung disease in Birt-Hogg-Dubé syndrome from those of tobaccorelated spontaneous pneumothorax. Histopathology 2014;64:741-9.

17. Gupta N, Seyama K, McCormack FX. Pulmonary manifestations of Birt-Hogg-Dubé syndrome. Fam Cancer 2013;12:387-96.

18. Min KY. Changes in the amounts of elastic and collagenous elements of normal aged and emphysematous lung: use of a model of static pressure-volume relationships. Nihon Kyobu Shikkan Gakkai Zasshi 1995;33:1161-7.

19. Reye RD, Bale P. Elastic tissue in pulmonary emphysema in Marfan syndrome. Arch Pathol 1973;96:427-31.

20. Duca L, Blaise S, Romier B, et al. Matrix ageing and vascular impacts: focus on elastin fragmentation. Cardiovasc Res 2016;110:298-308.

Cite this article as: Matsumoto T, Uto K, Oda H, Isaka T, Nagashima Y, Kanzaki M. Pleural changes in patients with pneumothoraces and Marfan syndrome. J Thorac Dis 2020;12(9):4877-4882. doi: 10.21037/jtd-20-926
21. Schlatmann TJ, Becker AE. Histologic changes in the normal aging aorta: implications for dissecting aortic aneurysm. Am J Cardiol 1977;39:13-20.

22. Carlson RG, Lillehei CW, Edwards JE. Cystic medial necrosis of the ascending aorta in relation to age and hypertension. Am J Cardiol 1970;25:411-5.

23. Bode-Jänisch S, Schmidt A, Günther D, et al. Aortic dissecting aneurysms - histopathological findings. Forensic Sci Int 2012;214:13-7.

24. Marsalese DL, Moodie DS, Lytle BW, et al. Cystic medial necrosis of the aorta in patients without Marfan's syndrome: surgical outcome and long-term follow-up. J Am Coll Cardiol 1990;16:68-73.

25. Walker CM, Rosado-de-Christenson ML, MartínezJiménez S, et al. Bronchial arteries: anatomy, function, hypertrophy, and anomalies. Radiographics 2015;35:32-49.

26. De Paepe A, Devereux RB, Dietz HC, et al. Revised diagnostic criteria for the Marfan syndrome. Am J Med Genet 1996;62:417-26.

27. Wanga S, Hibender S, Ridwan Y, et al. Aortic microcalcification is associated with elastin fragmentation in Marfan syndrome. J Pathol 2017;243:294-306.

28. Dyhdalo K, Farver C. Pulmonary histologic changes in Marfan syndrome. Am J Clin Pathol 2011;136:857-63. 